\title{
Apoyo social y violencia de pareja en estudiantes universitarias*
}

\section{Social Support and Intimate Partner Violence in University Students}

\author{
Rosa María Huerta Mata \\ (D) https://orcid.org/0000-0003-4661-5855 \\ Escuela Superior de Actopan \\ Universidad Autónoma del Estado de Hidalgo, México \\ huertamx@hotmail.com
}

Resumen: El objetivo de este artículo es analizar el proceso de vinculación-desvinculación del apoyo social que reciben algunas de las estudiantes universitarias que experimentan violencia de pareja. En los meses de septiembre y octubre del año 2017 se llevaron a cabo cuatro grupos focales de mujeres estudiantes de las licenciaturas en Derecho y Psicología en la Escuela Superior de Actopan, de la Universidad Autónoma del Estado de Hidalgo (UAEH), México. En la violencia de pareja que experimentan las estudiantes universitarias se involucran sus amigas como red de apoyo; sin embargo, el apoyo se fractura debido a que las víctimas no abandonan la relación de pareja, y por las amenazas de violencia que reciben las amigas por parte del agresor. Este trabajo contribuye al conocimiento sobre las subjetividades de las personas que apoyan a las jóvenes que presentan violencia de pareja.

* Este artículo se deriva de un estudio más amplio titulado "Apropiación del derecho a una vida libre de violencia: los(as) estudiantes universitarios(as)". Proyecto financiado por Prodep. Folio. UAEH-PTC-761.

cómo citAr: Huerta Mata, R. M. (2021). Apoyo social y violencia de pareja en estudiantes universitarias. Secuencia (110), e1851. DoI: https://doi.org/10.18234/secuencia.v0i110.1851

cC Esta obra está protegida bajo una Licencia Creative Commons Atribución-NoComercial 4.0 Internacional. 
Palabras clave: violencia de pareja; redes de apoyo; género; jóvenes; universidad.

Abstract: The objective of this article is to analyze the process of acceptance-rejection of the social support received by some of the university students who experience intimate partner violence. In september and october 2017, four focus groups of female law and psychology students were organized at the Escuela Superior de Actopan, part of the Autonomous University of the State of Hidalgo (UAEH), Mexico. In the partner violence that university students experience, their friends are involved as a support network. However, this support collapses either because victims do not leave the partner relationship, or because their friends are threatened with violence by the aggressor. The paper contributes to knowledge on the subjectivities of those who support young women experiencing intimate partner violence.

Keywords: intimate partner violence; support networks; gender; youths; university.

Recibido: 4 de mayo de 2020 Aceptado: 23 de junio de 2020

Publicado: 30 de abril de 2021

\section{INTRODUCCIÓN}

nivel internacional, los estudios sobre violencia de pareja en universita-
rios(as) son cuantitativos en su mayoría y predominan los que indagan
sobre las percepciones y las actitudes que en torno a este fenómeno desarro-
llan los(as) jóvenes (Aguanunu, 2014; Davidson, Lozano y Cole, 2015; Ferrer
et al., 2006; Kelmendi y Baumgartner, 2017; Li, Sun y Button, 2017; Nordin,
2019; Páramo y Arrigoni, 2018; Pugh, Li y Sun, 2018; Spencer, Morgan y Brid-
ges, 2017; Wang, 2019; Wilson y Smirles, 2020). Investigar sobre percepciones
y actitudes frente a la violencia de pareja en gran medida tiene la intención de
identificar a los predictores de las conductas violentas.
$\quad$ En México, $43.9 \%$ de las mujeres han sido agredidas en su última rela-
ción sentimental, o bien, por su actual esposo o pareja. De igual modo, son las 
jóvenes de entre 20 y 34 años quienes más experimentan la violencia de pareja (Instituto Nacional de Estadística y Geografía, 2019).

Respecto a la población de estudiantes universitarios(as), las investigaciones se centran en cuantificar el problema de la violencia en la pareja (Esquivel-Santoveña et al., 2020; Lazarevich, Irigoyen-Camacho y Velázquez-Alva, 2017; López-Cepero, Estrada-Pineda y Chan-Gamboa, 2018; Rodríguez, Riosvelasco y Castillo, 2018). Por su parte, los estudios cualitativos indagan las creencias y actitudes de los(as) jóvenes en torno a la violencia de pareja (Rojas-Solís, 2013), así como las experiencias de las jóvenes como víctimas de esta clase de violencia (Vázquez y Castro, 2008).

El presente trabajo surge de una particularidad encontrada en los testimonios de hombres y mujeres estudiantes universitarios(as), quienes participaron en una investigación más amplia, en la cual reportaron que, al presenciar situaciones de violencia de pareja dentro de la universidad, su respuesta por lo general es de indiferencia, y justifican su comportamiento con la idea de: "mejor ni te metas". Las razones son diversas, como el desinterés debido a que este tipo de violencia es común y se ha normalizado, por lo que, si intervienen, después la pareja se reconcilia y ellos(as) terminan involucrados(as) en un problema. No obstante, también identificamos discursos coincidentes en algunas de las participantes que nos permitieron analizar su respuesta crítica y de involucramiento cuando una de sus amigas sufre violencia en el noviazgo.

Matud Aznar y otros (2003) indican que, debido a que el agresor utiliza como estrategia de dominación el aislar de su núcleo social a la víctima, es entonces un área de intervención contra la violencia el restablecer la vinculación social de la víctima, para lo cual es esencial indagar sobre la manera en que se estructura el apoyo social. En concordancia, el objetivo del presente trabajo es analizar el proceso de vinculación-desvinculación del apoyo social que reciben algunas de las estudiantes universitarias que experimentan violencia de pareja.

\section{LA VIOLENCIA Y SUS ESTRUCTURAS}

Rita Segato (2003a) expone a la violencia como un hábito arraigado en la sociedad, el cual imposibilita que las personas puedan reconocerla y nombrarla, lo que la convierte en un fenómeno no reflexionado; por lo tanto, es "asimilado como parte de la 'normalidad' o, lo que sería peor, como un fenómeno 
'normativo', es decir, que participaría del conjunto de reglas que crean y recrean esa normalidad" (p. 132).

Segato (2003b) explica que las relaciones de género tienen un papel fundamental en el origen de la violencia. Asimismo, identifica que las estructuras elementales de la violencia se establecen a partir de dos ejes que se interconectan. Uno es el eje horizontal, el cual se encuentra constituido por relaciones de alianzas o de competición, y se sustenta en una idea de contrato entre iguales; es decir, en categorías sociales que se clasifican entre semejantes, sin embargo, se rige por el altercado. Por otro lado, el eje vertical se caracteriza por vínculos donde predomina la entrega o expropiación, y se sustenta en una idea de jerarquías que marcan un valor no igualitario entre quienes componen estos vínculos, de modo que es el estatus el que ordena las relaciones. Ambos ejes son cíclicos, se articulan y configuran un sistema. La autora indica que la articulación de los ejes es una economía simbólica; de manera que, el estar en un estatus menor se deriva de una expropiación simbólica, lo que también denomina extracción de plusvalía simbólica.

Actualmente, la doble inserción de la mujer en esta estructura, tanto en el eje vertical como en el horizontal, produce inestabilidad; por lo tanto, el sistema depende de la "dominación del hombre, que recurre cíclicamente a la violencia psicológica, sexual o física", con el fin de restaurar y reciclar el orden de estatus. Debido a todo lo anterior, Segato (2003b) propone el ámbito de la intimidad como como punto clave para redirigir políticas que pretenden eliminar la violencia.

\section{REDES DE APOYO SOCIAL}

Las redes de apoyo social permiten a los individuos establecer vínculos de ayuda y pueden constituirse de dos maneras: formal e informal. Las redes formales son "conformadas por organizaciones del sector público en los diferentes niveles de gobierno (federal, estatal y municipal) (Gobierno de México, Instituto Nacional de las Personas Adultas Mayores, s. f.). Por su parte, las redes informales surgen del ámbito privado de las personas; Lomnitz (1990) identifica que estas redes actúan de manera paralela o por debajo de las redes formales.

$\mathrm{Al}$ respecto, se ha encontrado que en México las mujeres víctimas de violencia por parte de su pareja recurren a redes de apoyo informales, por lo 
que son los(as) amigos(as) el apoyo social que las víctimas perciben como el más representativo (Estrada, Herrero y Rodríguez, 2012).

\section{VIOLENCIA DE PAREJA, SORORIDAD E IGUALDAD SUSTANTIVA}

La Organización de las Naciones Unidas (ONU) define la violencia por un compañero sentimental "como cualquier conducta por parte del cónyuge o de la pareja actual o una anterior que causa daño físico, sexual o psicológico" (onu. Mujeres, 2019).

En México existen leyes que protegen a las mujeres contra diversos tipos de violencia. La Ley de Acceso de las Mujeres a una Vida Libre de Violencia para el Estado de Hidalgo, en su artículo 5, fracción VIII, define a la violencia en el noviazgo como: "el acto abusivo de poder u omisión intencional, dirigido a dominar, someter, controlar o agredir a las mujeres de cualquier edad, mediante la realización de uno o varios tipos de violencia, durante o después de una relación de noviazgo, una relación afectiva o una relación sexual, y que viola sus derechos humanos". ${ }^{1}$

A pesar de la tipificación por ley de la violencia en el noviazgo, las dinámicas sociales crean una brecha entre los derechos de las víctimas y la aplicación efectiva de la ley. En este sentido, es fundamental analizar el apoyo social que pueden obtener las jóvenes que reciben este tipo de violencia.

En la teoría de género la sororidad es un espacio político, es la alianza entre mujeres en una relación de confianza y de apoyo, con el objetivo de efectuar modificaciones en sus vidas y en su contexto, de modo que este les sea más justo (Lagarde, 2006). En el ejercicio de la sororidad "no hay jerarquía, sino un reconocimiento de la autoridad de cada una. Está basado en el principio de equivalencia humana, igual valor entre todas las personas". ${ }^{2}$ Por lo tanto, la sororidad "reconstruye a las mujeres y es un camino real para ocupar

${ }^{1}$ Ley de Acceso de las Mujeres a una Vida Libre de Violencia para el Estado de Hidalgo, Periódico Oficial. Gobierno del Estado de Hidalgo, Poder ejecutivo, 2007, p. 4. Recuperado de http://www.congreso-hidalgo.gob.mx/biblioteca_legislativa/Leyes/12Ley\%20de\%20Acceso\%20 de\%20las\%20Mujeres\%20a\%20una\%20Vida\%20Libre\%20de\%20Violencia.pdf

2 R. M. Lagarde, "La política feminista de la sororidad", Mujeres en Red. El Periódico Feminista, 11 de julio de 2009. Recuperado de http://www.mujeresenred.net/spip.php?article1771 
espacios, lograr derechos, consolidar protecciones entre mujeres y eliminar el aislamiento" (Lagarde, 1997).

Por otro lado, desde el marco legislativo la igualdad sustantiva se define como: "el acceso al mismo trato y oportunidades para el reconocimiento, goce o ejercicio de los derechos humanos y las libertades fundamentales". ${ }^{3}$

Fredman (2016) indica que la igualdad sustantiva es compleja, por lo que no debe reducirse a la igualdad de oportunidades. Así, cuestiona que el derecho a una igualdad sustantiva esté delimitado a la dignidad o a la igualdad de oportunidades y resultados, para lo que propone una aproximación multidimensional. Explica que la igualdad sustantiva está dirigida hacia los grupos que muestran desventajas, las cuales implican no sólo la mala distribución de los recursos, también son las constricciones que, en función al estatus de los individuos, las estructuras del poder les imponen, de ahí que el trato igualitario para los individuos con diferentes constricciones puede resultar en desventajas. "Una de las funciones del derecho a la igualdad sustantiva es, por lo tanto, redirigir las desventajas para remover los obstáculos y lograr una elección genuina [...] Es necesario asumir que la elección en sí misma puede ser problemática, pues las personas frecuentemente adaptan sus decisiones a sus circunstancias" (Fredman, 2016, pp. 729-734) (traducción mía).

La misma autora afirma que la reorientación de las desventajas no debe contemplarse como la única dimensión de la igualdad sustantiva sobre la cual se requiere intervenir, también es necesario considerar los estigmas; es decir, las personas no acceden a veces al ejercicio de sus derechos para no ser estigmatizadas (en este caso, evitan reconocerse como víctimas de violencia de pareja para soslayar los juicios hacia ellas). Otra dimensión de la igualdad sustantiva consiste en tomar en cuenta que las personas requieren representación política en la toma de decisiones. Por último, una cuarta dimensión es la transformativa, en la cual se plantea que los individuos no deben ser quienes se acomoden a la estructura, sino que esta requiere cambiar para ellos, por lo que es necesario el debate.

La violencia de pareja es un fenómeno social complejo, lo que obliga a estudiarla desde las dinámicas sociales que establecen una brecha entre las víc-

${ }^{3}$ Ley de Igualdad Sustantiva entre Mujeres y Hombres en el Estado de Hidalgo, Periódico Oficial. Gobierno del Estado de Hidalgo, Poder ejecutivo, 2015, p. 6. Recuperado de http://www.con greso-hidalgo.gob.mx/biblioteca_legislativa/leyes_cintillo/Ley\%20para\%20la\%20Igualdad\%20 entre\%20Mujeres\%20y\%20Hombres\%20del\%20Estado.pdf 
timas y el ejercicio de sus derechos a través de la aplicación de la ley; es decir, es necesario aproximarnos a la comprensión de los obstáculos para alcanzar la igualdad sustantiva. Lo anterior pretende plantearse en este trabajo a través del análisis de las acciones de sororidad desarrolladas por las estudiantes universitarias cuando una compañera o amiga presenta violencia de pareja.

\section{MARCO CONTEXTUAL}

De entre las 32 entidades federativas de México, Hidalgo es una de las que registra alto rezago social, $50.6 \%$ de la población vive en pobreza, del cual $40.6 \%$ se encuentra en pobreza moderada y $8 \%$ en pobreza extrema. ${ }^{4}$ En algunos municipios del Valle del Mezquital la pobreza alcanza a $65 \%$ de la población (Gobierno de México. Secretaría de Bienestar, 2019).

En el Valle del Mezquital la identidad cultural se centra en el grupo indígena hñahñú. Esta zona se caracteriza por una orografía árida, en consecuencia, se ha estimulado la conformación de tierras de cultivo por medio de la creación de uno de los sistemas de riego con aguas negras más grande del mundo; sin embargo, unos pocos acaparan el acceso a este suministro, lo que genera severos problemas de desigualdad económica y social, y obliga a la migración para laborar en Estados Unidos de América (Arzate, 2011).

En este contexto, desde hace 20 años la Universidad Autónoma del Estado de Hidalgo (UAEH) ofrece educación superior a través de la Escuela Superior de Actopan. Actualmente la institución brinda las carreras de Psicología, Diseño Gráfico, Creación y Desarrollo de Empresas y Derecho. A la institución acuden jóvenes provenientes de comunidades rurales y semirrurales aledañas.

\section{METODOLOGÍA}

Esta es una investigación cualitativa. En los meses de septiembre y octubre de 2017 se llevaron a cabo cuatro grupos focales de mujeres estudiantes de las

${ }^{4}$ L. Naranjo, "Hidalgo: 50.6\% vive en la pobreza", El Independiente de Hidalgo, 1 de febrero de 2019. Recuperado de https://www.elindependientedehidalgo.com.mx/hidalgo-50-6vive-en-la-pobreza/ 
licenciaturas en Derecho y Psicología de la Escuela Superior de Actopan, de la UAEH. Ambas licenciaturas se encuentran en un mismo centro universitario, lo cual permite abordar un contexto común en la población de estudio. Se eligió trabajar en las licenciaturas con mayor porcentaje de mujeres en su matrícula escolar, Derecho (60.4\%) y Psicología (83.6\%) (Universidad Autónoma del Estado de Hidalgo, 2017).

La técnica de grupo focal consiste en generar la conversación sobre un tema de investigación con la finalidad de obtener información sobre los pensamientos, sentimientos y experiencias de los(as) participantes (Álvarez-Gayou, 2012). En este caso, se trabajó con grupos focales debido a que se pueden recolectar datos de un mayor número de voces en un menor tiempo, además de que es posible la sistematización de experiencias y la "reconstrucción participativa de saberes" (Franco y Orozco, 2019, p. 289). Pero una de las desventajas de utilizar esta técnica es que no es posible entrevistar a profundidad a cada uno(a) de los(as) participantes.

Se utilizó el modelo de entrevista a profundidad, la cual inició con el planteamiento del tema a conversar en el grupo focal: "hablemos sobre el ámbito de la relación de pareja y la violencia que pueden llegar a experimentar. ¿Qué situaciones viven las y los jóvenes de la Escuela Superior de Actopan respecto a la violencia en la pareja? Puedes platicar algo personal o algo que tú has observado en estudiantes que asisten a este campus de la universidad."

En los criterios para conformar la muestra se consideró que las participantes fueran mayores de 18 años, estudiantes regulares del turno matutino o vespertino, y que cursaran cualquier semestre de las licenciaturas de Psicología o Derecho. Participaron en total 28 mujeres, quienes asistieron de manera voluntaria y firmaron un consentimiento informado. La muestra se encuentra en el rango de 18 a 27 años, con un promedio de edad de 20 años; $89 \%$ son solteras, una es casada, dos viven en unión libre y cuatro de las participantes son madres.

Las entrevistas se llevaron a cabo en dos sesiones con una duración en promedio de dos horas cada una; todas fueron grabadas y transcritas. Con los datos obtenidos se realizó el análisis de contenido a través de la perspectiva de género y se establecieron las siguientes categorías: 1) la relación de las víctimas de violencia de pareja con los recursos institucionales -redes de apoyo formal y familiar-; 2) el apoyo social establecido por las compañeras de la víctima de violencia de pareja, y 3) vulneración del apoyo social de las compañeras hacia la víctima. 


\section{RESULTADOS \\ RECURSOS INSTITUCIONALES DE APOYO SOCIAL CONTRA LA VIOLENCIA EN LA PAREJA}

Los testimonios evidencian que las jóvenes, por experiencia propia o de sus amigas, no consideran a las instituciones gubernamentales como un lazo social para la protección de la violencia; es evidente la descalificación y desconfianza que ellas establecen respecto al servicio que reciben al momento de levantar una denuncian en contra de su agresor.

La verdad las instituciones no hacen caso, en una ocasión mi pareja me golpeó, me dejó el ojo hinchado, entonces yo voy, lo demando, voy con el médico para que me examinara y me dice: "ah, no es nada". Le digo: "o sea, ¿tengo que venir casi muriéndome para que me hagan caso? ¿iQué onda!?” Y no lo detuvieron, no pasó nada, simplemente no pasó nada. Entonces creo: ¿para qué voy a esas instituciones si no hacen nada? No sirve, para mí no sirve (Grupo focal matutino de mujeres estudiantes de Psicología).

Las estudiantes muestran desconfianza hacia las instituciones debido a que experimentan la ineficacia en el respeto de sus derechos humanos básicos, pues en estos contextos se reproduce el culparlas y responsabilizarlas de la violencia, además de que minimizan las agresiones que denuncian; por lo tanto, las participantes perciben a las instituciones como indiferentes ante sus demandas de apoyo y protección. Asumen que denunciar las expone a respuestas más violentas por parte del victimario. Es así como se configura un abandono institucional y la reproducción de violencia de género contra las mujeres jóvenes.

Otro lazo social que no establecen las univeristarias para visibilizar su situación es cuando evitan comunicar a sus familias que sufren violencia de pareja: "igual pues es la vergüenza de decir: '¡ay!, ¿cómo les voy a decir a mis papás que estoy siendo violentada?' Se van a enojar conmigo, o cosas así, de lo que yo he escuchado [que dicen] mis compañeras o mis amigas, es por eso, por su orgullo tonto, por la vergüenza" (Grupo focal matutino de mujeres estudiantes de Psicología).

Para algunas estudiantes, tanto las instituciones gubernamentales como sus familias representan mecanismos que reafirman el eje vertical de las estructuras de la violencia, por lo que evitan acudir a estas redes de apoyo para protegerse de lo que, en términos de Segato (2003b), les representaría una mayor expropiación de plusvalía. 
$\mathrm{Al}$ parecer la violencia se experimenta en secreto, pero en la cotidianidad de las aulas y el contexto universitario también se suscitan eventos de violencia de pareja, fenómeno que es ejercido y atestiguado por los(as) estudiantes. En el caso de las participantes de esta investigación, se manifiesta la restricción de compartir su situación con las instituciones y las familias; por ello, en el espacio universitario, las amigas se convierten en el vínculo más fuerte para enfrentar la violencia de pareja.

\section{EL APOYO SOCIAL ANTE LA VIOLENCIA DE PAREJA EN LA UNIVERSIDAD}

Qué habría sido de las mujeres en el patriarcado sin el entramado de mujeres alrededor, a un lado, atrás de una, adelante, guiando el camino, aguantando juntas. ¿Qué sería de nosotras sin nuestras amigas? ¿Qué sería de las mujeres sin el amor de las mujeres? (Lagarde, 2006, p. 124).

"A nivel mundial en 2012, en uno de cada dos casos de mujeres asesinadas, el autor era su compañero sentimental" (onU. Mujeres, 2019). México se ha convertido en uno de los 20 países más peligrosos para una mujer. ${ }^{5} \mathrm{Al}$ respecto, entre los años 2015 y 2018 se duplicaron los feminicidios; en 2015 ocurrieron 407, mientras que en 2018 la cifra fue de $845 .^{6}$ Actualmente, en el país suceden 10.5 feminicidios por día, ${ }^{7}$ por lo que la Organización de las Naciones Unidas ha colocado a México en el primer lugar en feminicidos en América Latina. ${ }^{8}$

5 "México, entre los peores 20 países para ser mujer", Forbes Woman, 7 de febrero de 2019. Recuperado de https://www.forbes.com.mx/mexico-entre-los-20-peores-paises-para-ser-mujer/ ?fbclid=IwAR3tQySjiOOKaeG5mO6cFnAMWUzzX9VSEK3BjjNasKcv3bMmUGefSUxFr34

${ }^{6}$ A. Langner y E. Olivares, "Cifras oficiales: la violencia contra las mujeres sigue al alza", La Jornada, 8 de marzo de 2019. Recuperado de https://www.jornada.com.mx/ultimas/politi ca/2019/03/08/cifras-oficiales-la-violencia-contra-las-mujeres-sigue-al-alza-9689.html

${ }^{7}$ J. Xantomila, "ONU: Feminicidios en México crecieron diariamente de 7 a 10 en tres años", La Jornada, 5 de marzo de 2020. Recuperado de https://www.jornada.com.mx/ulti mas/sociedad/2020/03/05/onu-feminicidios-en-mexico-crecieron-de-7-a-10-diarios-en-tres-a nos-8647.html

${ }^{8}$ L. Gómez, "En feminicidios México es el primer lugar en A L: magistrada", La Jornada, 3 de marzo de 2019, Recuperado de https://www.jornada.com.mx/ultimas/2019/03/06/en-femi nicidios-mexico-es-primer-lugar-en-al-magistrada-4285.html 
En el estado de Hidalgo, de acuerdo con información del Secretariado Ejecutivo del Sistema Nacional de Seguridad Pública (SESNSP), en 2019, la entidad, de entre las 32 del país, se ubica en el sitio número once en la ocurrencia

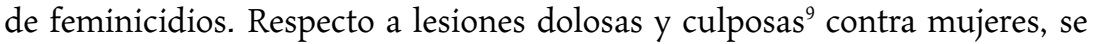
ubica en el séptimo y sexto lugar, respectivamente. ${ }^{10}$

En relación con el feminicidio, Lagarde (2006) señala que "no es sino la punta del iceberg de formas de violencia de género integradas a la convivencia, recreadas por la impunidad, hechos extremos de la opresión patriarcal" (p. 128). En este sentido, es relevante analizar los elementos invisibles del iceberg que se configuran en la vida cotidiana de las universitarias, como lo es la violencia de pareja. ${ }^{11}$ En el presente estudio analizamos las diversas formas en las que las jóvenes se involucran en sororidad con las compañeras violentadas.

Mi novio me decía: "es que ya no les hables, es que esa persona nada más te sonsaca, a mí no me gusta que te juntes con ellos". Él como que se molestaba y ya empezaba a jalonearme, a pellizcarme, y yo pues me dejaba, no decía nada y lo único que hacía era llorar. [En el aula] siempre nos sentábamos hasta atrás y ahí era donde de cierta forma yo pensaba que pasábamos desapercibidos, pero pues los que estaban a nuestro alrededor sí se daban cuenta, yo a veces sí escuchaba [comentarios hechos por los(as) compañeros(as)], es que ya se

${ }^{9}$ Artículo $8^{\circ}$ : Las acciones $\mathrm{u}$ omisiones delictivas solamente pueden realizarse dolosa o culposamente.

Artículo $9^{\circ}$ : Obra dolosamente el que, conociendo los elementos del tipo penal, o previendo como posible el resultado típico, quiere o acepta la realización del hecho descrito por la ley, y Obra culposamente el que produce el resultado típico, que no previó siendo previsible o previó confiando en que no se produciría, en virtud de la violación a un deber de cuidado, que debía y podía observar según las circunstancias y condiciones personales (Código Penal Federal, 2019).

${ }^{10}$ A. Hernández, "Hidalgo, 11 en feminicidios", El Independiente de Hidalgo, 6 de marzo de 2019. Recuperado de https://www.elindependientedehidalgo.com.mx/hidalgo-11-enfeminicidios/

${ }^{11}$ Investigaciones recientes se centran en la intervención educativa para modificar actitudes y creencias ante la violencia, o bien, generar habilidades en los(as) universitarios(as) para identificar situaciones de violencia en la pareja y para responder de manera efectiva cuando son partícipes o espectadores, pues es común que la negación, minimización o el no darle importancia al evento puedan impedir el que reconozcan la violencia e intervengan para limitarla o pararla (Amar et al., 2015; Weisz et al., 2007). Lo anterior, ha sido abordado a partir de situaciones hipotéticas (Ruiz, Expósito y Bonache, 2010), o por la medición de las reacciones que manifiestan las personas que apoyan a las víctimas al momento en el que les cuentan las situaciones de violencia (Weisz et al., 2007). 
pelearon ellos dos, es que ya la hizo llorar... (Grupo focal vespertino de mujeres estudiantes de Psicología).

En la universidad se instaura la violencia de pareja como un proceso de interacciones habituales dentro del aula, dinámica tolerada por el grupo de compañeros(as) que se involucran como espectadores(as). Sin embargo, algunas jóvenes muestran responsabilidad para intervenir, por lo tanto, de espectadoras pasan a un papel más activo, se acercan a la víctima, la proveen de apoyo sin que lo solicite, le aconsejan y le cuestionan el trato indigno que recibe por parte de su pareja, procuran impulsar en ella la reflexión sobre la violencia que experimenta, al igual que de convencerla de terminar con la relación. De esta manera, generan protección grupal y emprenden acciones de sororidad al visibilizar la violencia física y psicológica que recibe la compañera, labores que trastocan la dependencia de la joven hacia su agresor; por lo tanto, es a través del grupo de amigas que la víctima adquiere un nivel de autonomía frente a la violencia perpetrada por su pareja.

[...] ahorita tengo una amiga que tenía un novio que la trataba mal, no podía estar ella sola porque ya cuando veíamos ya andaba aquí cuidándola, andaba rondando por toda la escuela. Ella llegaba con unos moretones horribles [...] entonces terminó con él, se la quitamos, porque se la quitamos, la tuvimos mucho tiempo con nosotras, tratamos de que estuviera muy apegada con nosotras (Grupo focal matutino de estudiantes de Derecho).

Las jóvenes manifiestan sororidad al empatizar con las víctimas, no las asustan o amenazan al aconsejarlas que abandonen la relación de pareja. Asimismo, al ejercer la sororidad las estudiantes vulneran el aislamiento de la víctima y la dotan de poder para hacer valer su derecho a una vida libre de violencia. Así, en grupo cuestionan y confrontan la violencia de pareja, desmarcan a la compañera del trato cosificador que recibe de su novio; ella, al protegerse de la violencia, rompe con el orden jerárquico de la estructura que la posiciona en subordinación, así, limita lo que Segato (2003b) establece como la expropiación simbólica, de manera que su respuesta de protección le permite configurar plusvalía simbólica.

Lagarde indica que la sororidad implica "una responsabilidad ética por parte de quienes tienen más para construir la ciudadanía de género" (Lagarde, 2006, p. 131). En este sentido, las amigas, al contar con mayor autonomía 
frente al agresor, apoyan a la víctima, y entre todas ejercen su ciudadanía en el espacio universitario. Por otro lado, la sororidad es también una "alianza existencial y política, cuerpo a cuerpo, subjetividad a subjetividad con otras mujeres, para contribuir con acciones específicas a la eliminación social de todas las formas de opresión" (Lagarde, 2006, p. 126). Las amigas resguardan a la joven de la violencia, y para ello involucran al cuerpo como medio de protección, también comparten subjetividades. Esto último sin alcanzar una reflexividad con orientación política, por lo que estas mujeres ejecutan esfuerzos para configurar una vida libre de violencia, pero sin plantearlos en términos de derechos. De igual modo, son acuerdos de protección y supervivencia carentes de objetivos dotados de un sentido colectivo, labores aisladas, así como pactos solidarios de defensa sin apoyo institucional.

Lagarde (2013) indica que para que se presente sororidad es necesario la configuración de intereses comunes entre las mujeres; en este caso, el oponerse a la violencia de género en la pareja es un estímulo para la sororidad. El grupo de amigas confía en que, al intervenir en la violencia de pareja sus estrategias tendrán el efecto de alejar a la joven del agresor. Por lo tanto, asumen la responsabilidad de proteger a la víctima y creen que sus respuestas de apoyo serán eficaces para lograr eliminar la violencia de pareja.

Una compañera de cuarto tuvo problemas con su novio, y en una de esas la chava venía corriendo de la carretera a mi casa, desesperada, con todo y sus maletas, ${ }^{12}$ porque su digamos marido ya, la empezó a corretear porque la iba a golpear, nada más porque saludó a alguien. Cuando voy viendo que venía el otro, abrimos la puerta de la casa, y yo cerré, nos metimos al cuarto y le dije: "tranquilízate, no va a pasar nada, pero dime iqué está pasando?", "-Es que me quiere pegar". Y le dije, "yo te puedo ayudar siempre y cuando tú te dejes, si me ves que yo lo llego a golpear porque me estoy defendiendo y tú agarras y te atraviesas y evitas que lo golpee, no te puedo ayudar, solamente yo me estoy protegiendo" (Grupo focal matutino de mujeres estudiantes de Derecho).

En ocasiones, al intervenir en la defensa, las amigas se exponen a recibir violencia física por parte del agresor. Además, las respuestas de las jóvenes

${ }^{12}$ Los(as) universitarios(as) por lo general comparten la renta de un cuarto con otros(as) estudiantes, los fines de semana es común verlos(as) regresar de sus comunidades o municipios con sus maletas, tras pasar el fin de semana en casa de sus familiares. 
son de no tolerar ni naturalizar la violencia de pareja, así que la cuestionan, tratan de frenarla. Es probable que las compañeras que se solidarizan con las víctimas también vivan violencia de pareja, pero es evidente el rechazo que muestran hacia la violencia extrema. Por otro lado, tal vez respondan en función de su experiencia, pues, según Sundborg et al. (2015), las personas que han vivido violencia dudan menos en prestar ayuda.

Los resultados del apoyo que las víctimas reciben de sus amigas no siempre son los de desvincularlas totalmente de la pareja, muchas jóvenes permanecen o regresan a las situaciones de violencia, de modo que buscan ayuda, pero no abandonan la relación $;^{13}$ razón por la cual sus compañeras y/o amigas deciden ya no apoyarlas o hacerlo en menor medida, y así salir de la participación que adquieren en la relación de violencia de pareja.

\section{SE VULNERA EL APOYO SOCIAL DE LAS COMPAÑERAS HACIA LA VÍCTIMA}

Las compañeras y/o amigas se involucran con el problema de la violencia de pareja al mostrar interés y desarrollar mecanismos de protección para la víctima. El objetivo de esta red de apoyo informal es detener la violencia, pero al no lograrlo, se frustra su ayuda, situación que genera la desvinculación social de la víctima. La práctica del derecho a una vida libre de violencia -al menos la pretensión de su adquisición- por parte de las jóvenes, se convierte en un ideal al enfrentarse con el sabotaje del victimario, pues las compañeras pasan de intermediaras a víctimas.

Una amiga se peleaba mucho con su novio y yo a veces me metía y les decía: "iya no se peleen! Estamos en la escuela". Y una vez su novio me dijo: "pues no te metas, son problemas de nosotros". Entonces como que de ahí dije: "bueno, buen punto, o sea, no sé por qué me tengo que meter [...] Entonces ahí decides quedarte como fuera, para no estar peleándote con tu amiga, con el novio de

${ }^{13}$ Existen diversas teorías que tratan de explicar las causas por las que las jóvenes permanecen en relaciones de pareja violentas, como es el amor romántico (Adams, Williams y Nagoshi, 2015), la falta de identificación de la violencia por parte de la víctima (Páramo y Arrigoni, 2018), la protección del agresor por parte de la víctima (Morales y Rodríguez, 2016), y la falta de recursos que respalden la seguridad de la víctima al abandonar a su pareja (López-Cepero et al., 2018). 
tu amiga. Hasta nada más escucharla, nada más saber por qué estaban peleando, pero pues hasta ahí (Grupo focal matutino de mujeres estudiantes de Psicología).

En este caso, el agresor censura la sororidad entre las compañeras y su víctima, con la finalidad de ajustar sus relaciones a principios sociales de represión, de no cuestionamiento ni confrontación de su conducta agresiva, de manera que la relación de la víctima con sus amigas se sujeta a las alianzas subrepticias, acalladas en el espacio público y sólo ejercidas en lo privado.

En consecuencia, se produce una brecha entre ellas, lo cual es ambiguo. Por un lado, algunas se distancian de la víctima y mantienen un vínculo como espectadoras, aunque no indiferentes, pues se muestran preocupadas por el bienestar de la joven, conservan un grado de responsabilidad con la víctima, pero su postura ya no es tan activa.

Otras veces, el apoyo de las amigas hacia la víctima pasa de brindar protección al resguardarla y distanciarla del victimario, así como de la confrontación directa con él, a un apoyo privado, velado y limitado a la escucha. No obstante que, con frustración, permanece la sororidad en la escucha y en el interés por la compañera. "Hay una chica en mi salón que vivió violencia muy fea, el chico ahora nos ve feo a las que le hablamos, incluso traje a mi hija [a la universidad] y dijo: ‘jay!, ¿cómo traen a sus pinches chamacos a la escuela?' Y ahí estaba mi hija. Nada más porque soy amiga de ella, ya también decide tomarla conmigo" (Grupo focal matutino de mujeres estudiantes de Psicología).

En la vida universitaria el agresor llega a valerse de recursos no verbales como las miradas de enojo o amenazantes hacia las compañeras, o de expresiones verbales que realiza en voz alta en espacios comunes del centro escolar, para amedrentar a la red de apoyo, controlar la interacción social de su pareja y marcar su aislamiento. Ante el miedo que genera el abusador, para la red de apoyo el costo de ayudar a sus amigas es muy alto, al grado de arriesgar su integridad física y la de sus hijos(as). En algunos casos, las amigas retiran la ayuda debido a factores contextuales, por presión de su familia de origen o de su pareja, para que resguarde su seguridad.

Por otro lado, las amigas no se identifican plenamente como víctimas colaterales de la violencia de pareja en la universidad, tampoco manifiestan sentimientos de injusticia ni generan prácticas colaborativas para el reconocimiento de sus derechos. No obstante, se convierten en covíctimas de la violencia de pareja a través de las amenazas que reciben del agresor. Además, no 
plantean a la amenaza como un delito, a pesar de estar tipificado en el código penal mexicano.

Una de mis compañeras una ocasión se acercó conmigo y me dijo: "pues yo veo que te trata muy mal, pero a mí me da miedo acercarme a tu novio porque siento que me va hacer algo". Y de hecho sí, porque después me comentó que mi novio le había dicho que ya no se acercara a mí, que me dejara de hablar. Fue la única persona de la que yo supe que realmente se acercó o que tenía tal vez un interés en ayudarme, de cierta forma, pero sí me dijo que por miedo mejor no hacía nada... (Grupo focal vespertino de mujeres estudiantes de Psicología).

En algunos casos las amigas retiran la ayuda en respuesta al miedo que les genera la prohibición por parte del agresor -de manera verbal- de interactuar con la víctima. Cuando el abusador extiende la violencia hacia el grupo de apoyo se determina con mayor impulso la inequidad, y los esfuerzos por restituir la dignidad de las víctimas de violencia de pareja resultan mermados. Aquí proponemos que se aplica lo que Lagarde (2012) determina como la escisión del género "este extrañamiento entre mujeres: aquellas barreras infranqueables que las distancian hasta el grado de impedirles reconocerse e identificarse". En este caso, el extrañamiento procede a una fase de sororidad y es el resultado de la violencia, del miedo y la frustración que genera en las jóvenes. Por otro lado, la red de apoyo, a pesar de alejarse de la víctima, no pierde su sentido crítico sobre la situación; es decir, no crean una distancia crítica, sin embargo, ahora la hace respecto al victimario y la extienden a la víctima.

Ya ni si quiera la quiero escuchar, sí me importa y la quiero, pero es que la escucho y me frustro porque yo le digo: "no seas ridícula, ahorita vienes y chillas y después vas a estar ahí con él, no me hagas perder el tiempo". Y ya ni si quiera por chisme dan ganas de escuchar, porque es lo mismo por lo que se pelean siempre, ya hasta me aburre, cuando veo que me va a contar toda su tristeza yo me quedo frustrada, porque no puedo hacer nada y al rato los ves otra vez besándose (Grupo focal matutino de mujeres estudiantes de Psicología).

Las amenazas de violencia resquebrajan la sororidad entre las compañeras, quienes excluyen a la víctima para evitar ser objeto de violencia, y también establecen distancia debido a que la joven permanece en la relación de pareja y no cumple con la expectativa de lograr que el apoyo de sus amigas 
devenga en la disolución de la relación. Justo su condición de víctimas genera vínculos de ayuda; sin embargo, su vigencia no es amplia, pues el que permanezcan como víctimas motiva la fractura de su red. Lógica que reduce las respuestas de apoyo, se deprecia la utilidad de la ayuda y se genera un sinsentido de la sororidad, situación que resulta en una jerarquía entre el grupo y la víctima; ella ahora es la "otra", la que persiste en la violencia de pareja, y así, la joven pierde valor frente a su red de apoyo.

En consecuencia, a la violencia de pareja las amigas ya no la asumen como un problema común, se deslindan de la responsabilidad de apoyar y proteger, de modo que se establece la diferencia entre ellas y "las otras", las que fastidian, de las que se cansan, a quienes culpan de permanecer en la relación de violencia. Así, la víctima se posiciona en desigualdad frente a la pareja y el resto de sus compañeras, con un riesgo mayor de exclusión social, pues "el aislamiento de las víctimas de las fuentes de apoyo social se ha asociado con un aumento en la severidad de abuso de pareja" (García et al., 2014, p. 249). En este sentido, ya habían obtenido una red de apoyo con las amigas (para algunas la única), y al permanecer o regresar con el agresor, se debilita o se pierde el apoyo, el aislamiento se vuelve más marcado -al estar desprovistas de una red-, entonces es probable que se encuentre más vulnerables a la violencia extrema.

Cuando el agresor lograr distanciar a las amigas se produce la desigualdad frente al hombre y la desigualdad entre ellas se instituye, además de que se configura una estructura de violencia más compleja que enmarca la segregación social de este grupo de estudiantes en el contexto universitario. En dicha dinámica se devela el eje vertical de la estructura de la violencia el cual, explica Segato (2003a), se configura a partir de un sistema de estatus que consiste en el poder que se le quita (usurpa) a la mujer para asegurar su sumisión y lograr el dominio de los hombres. En la actualidad, las mujeres se insertan en este orden jerárquico y generan autonomía, lo que las lleva a adquirir una "posición híbrida" dentro del sistema. La no correspondencia de la posición de las mujeres en este sistema con sus subjetividades genera violencia para aprisionarlas en la subordinación.

Asimismo, la sororidad les permite a las estudiantes adquirir la "posición híbrida", por ello reciben la violencia que las lleva a mantenerse en la subordinación, posición que es reforzada cuando las compañeras establecen un valor no igualitario entre ellas y la víctima, con la intención de que el estatus les permita ordenar las relaciones (además de protegerse de la violencia); 
sin embargo, como todas se encuentran en un nivel jerárquico menor, estas acciones conllevan al respaldo en la extracción de plusvalía simbólica de la víctima y de las universitarias en general.

Por lo tanto, las estudiantes universitarias, debido a la violencia de pareja, participan en el ámbito público en condiciones de desigualdad, tanto entre ellas como con los hombres que las violentan, pues la violencia de pareja en el contexto universitario conlleva a que algunas jóvenes, de manera individual y grupal, acumulen desventajas que las desafilian, lo que limita la trascendencia de su esfuerzo por construir sus derechos humanos, pues no logran acceder en su totalidad a las condiciones que los garanticen, así pues, la violencia de pareja en universitarias ostenta una repercusión comunitaria.

Centrar la atención en el proceso de vinculación-desvinculación entre la víctima y su red de apoyo social en la universidad es clave para contribuir al bienestar y prevenir que la violencia se torne más severa contra todas las jóvenes involucradas. Como menciona Fredman (2016), la igualdad sustantiva requiere abordarse de manera multidimensional, de esta forma, las jóvenes que son la red de apoyo, al pretender reorientar las desventajas de la víctima, tendrían que contar con respaldo, y justo en esta fase se habrían de incorporar mecanismos de protección más amplios como la información y la interacción de instancias gubernamentales e institucionales con las amigas y la víctima. Por lo tanto, la dimensión transformadora de la igualdad sustantiva será efectiva si se producen cambios estructurales en favor de las personas en desventaja.

\section{DISCUSIÓN}

Este estudio tiene el objetivo de contribuir al conocimiento sobre las redes de apoyo de las jóvenes universitarias que presentan violencia de pareja, en específico, para visibilizar el proceso de apoyo que las compañeras universitarias configuran para la víctima.

Los resultados de este trabajo indican que las estudiantes universitarias muestran desconfianza hacia las instituciones. En una investigación efectuada en México con mujeres jóvenes se encontró que son pocas las que denuncian a su pareja cuando reciben violencia, y quienes denunciaron ante las autoridades no obtuvieron la respuesta de proceder con la aplicación de la legislación correspondiente (González y Fernández de Juan, 2010). Además, son revictimizadas (Frías y Agoff, 2015). 
Por otro lado, las jóvenes no confían a sus familiares que experimentan violencia de pareja debido a que tienen vergüenza, lo que coincide con lo encontrado por Rodríguez, Riosvelasco y Castillo (2018). Asimismo, en una investigación mixta realizada con 64 mujeres mexicanas de 20 a 65 años, se identificó que $56.1 \%$ de las víctimas no buscaron apoyo en sus familias. Y las que recurrieron a la familia experimentaron vergüenza, ya que se le atribuyó la responsabilidad de la violencia a su elección de pareja (Frías y Agoff, 2015).

Lo encontrado en esta investigación es similar a lo expuesto por López-Cepero, Estrada-Pineda y Chan-Gamboa (2018) en cuanto a que las amistades son percibidas como una de las más fuertes redes de apoyo para las personas víctimas de violencia. En una investigación sobre violencia de pareja en alumnos(as) universitarios(as) realizada en Chile, se encontró que "respecto a la búsqueda de ayuda 57\% mantuvo esta situación en secreto, quienes compartieron este problema, lo hicieron en mayor número con los(as) amigos(as) (34\%), y en menor medida con sus familiares (12.6\%)" (Vizcarra y Póo, 2011, p. 94). Dunham y Sen (citados por Weisz et al., 2007), en un estudio con 182 mujeres universitarias quienes vivieron violencia de pareja, identificaron que $67 \%$ de ellas reveló primero su situación a sus amigos.

Las redes de apoyo de las compañeras universitarias motivan a las mujeres víctimas de la violencia de pareja a abandonar la relación, lo que es coincidente por lo encontrado por Edwards, Dardis y Sylaska (2015). Asimismo, en algunos casos, las compañeras proveen a la víctima de apoyo sin que lo solicite y la motivan para buscar ayuda, esto es similar a los resultados de Stylianou, Counselman-Carpenter y Redcay (2018).

La respuesta de las amigas es la de proteger a la víctima; sin embargo, el miedo que les produce las amenazas del agresor las lleva a retirar el apoyo. $\mathrm{Al}$ respecto, Weisz y otros (2007) encontraron que cuando la severidad de la violencia es alta, la evitación por parte de la red de apoyo es la conducta más usual, fenómeno que puede responder al miedo que los pares presentan ante la persona abusadora. Por otro lado, si la violencia es severa es más común que los amigos manifiesten respuestas de evitación (Michel y Hudson, 1983. Citado en Weisz, et al., 2007).

Algunas limitaciones del presente estudio deben ser reconocidas. Nuestros datos se suscriben a estudiantes universitarias que pertenecen a una región específica de México. No es un estudio longitudinal, por lo que se desconoce el proceso de apoyo a largo plazo. Futuros estudios pueden replicarse en población no heterosexual, no universitaria y de otras regiones del país. 


\section{CONCLUSIONES}

En este trabajo se desarrolla el análisis del proceso de vinculación-desvinculación del apoyo social que reciben algunas estudiantes que experimentan violencia de pareja en la universidad. En el proceso de apoyo informal se involucran las amigas de la víctima, quienes desarrollan el papel de red de apoyo contra la violencia. Sin embargo, la ayuda se fractura cuando la red percibe como frustrado el que la joven abandone al agresor, y como resultado de las amenazas de violencia contra ellas y/o sus hijos(as) por parte del victimario, así, la sororidad del grupo de apoyo pasa de la empatía a la evitación de la víctima. Como consecuencia, las jóvenes acumulan desventajas en su trayecto por la universidad, originadas por recibir violencia por parte de su pareja y de la consecuente desvinculación con su red de apoyo. Por tanto, el proceso de apoyo social hacia la víctima se asocia a la recuperación de plusvalía simbólica y a la desvinculación entre la víctima y la red de apoyo dentro de la universidad, a la expropiación de plusvalía simbólica. No obstante, esto último ya no sólo es por parte de los agresores, sino que también de las compañeras que eran su red de apoyo. Debido a que la estructura de la violencia es cíclica, dicha dinámica deja a las víctimas en una situación de mayor subordinación social.

Es prioritario dirigir acciones que mejoren la confianza de las mujeres hacia las instituciones, acciones que consideren crucial el que las redes de apoyo formal contemplen e integren a las redes de apoyo informal que, en las amigas, tienen las víctimas, donde se priorice tanto la seguridad de la víctima como la de su red. Todo ello, como un medio para la inclusión social de las jóvenes universitarias de manera democrática en condiciones que no amenacen su integridad y que contribuyan a la conformación de la igualdad sustantiva. Por último, maestros(as), trabajadores(as) sociales, psicólogos(as) puede usar los resultados de este trabajo para aumentar esfuerzos dirigidos a modificar las dinámicas que impiden a las jóvenes universitarias mantener sus redes de apoyo ante la violencia de pareja.

\section{LISTA DE REFERENCIAS}

Adams, R. H., Williams, L. y Nagoshi, J. (2015). Help-seeking and help-offering for teen dating violence among acculturating Mexican American adolescents. 
Children and Youth Services, 53, 219-228. DoI: https://doi.org/10.1016/j.childyouth.2015.04.001

Aguanunu, R. I. (2014). Dating violence among university students: the role of gender and age. Research on Humanities and Social Sciences, 4(27), 19-22. Recuperado de https://www.iiste.org/Journals/index.php/RHSS/article/view/18424/18760

Álvarez-Gayou, J. L. (2012). Cómo hacer investigación cualitativa. (Fundamentos y metodología). México: Paidós Educador.

Amar, A., Tuccinardi, N., Heislein, J. y Simpson, S. (2015). Friends helping friends: a nonrandomized control trial of a peer-based. Nurs Outlook, 63(4), 496-503. DoI: https://doi.org/10.1016/j.outlook.2015.01.004

Arzate S. J. (2011). Región y desigualdades económico-sociales. El caso del Valle de Toluca y el Valle del Mezquital. Espacios Públicos, 14, 248-257. Recuperado de http://ri.uaemex.mx/handle/20.500.11799/39730

Código Penal Federal (2019). Justia. México. Artículo 7 al 11. Recuperado de https:// mexico.justia.com/federales/codigos/codigo-penal-federal/libro-primero/titulo-primero/capitulo-i/\#articulo-8

Davidson, M. M., Lozano, N. M. y Cole, B. P. (2015). Relations between intimate partner violence and forgiveness among college women. Journal of Interpersonal Violence, 30(18), 3217-3243. DoI: https://doi.org/10.1177/0886260514555008

Edwards, K. M., Dardis, C. M y Sylaska, K. M. (2015). Informal social reactions to college women's disclosure of intimate partner violence: Associations with psychological and relational variables. Journal of Interpersonal Violence, 30(1), 25-44. DOI: https://doi.org/10.1177/0886260514532524

Esquivel-Santoveña, E. E., Rodríguez, R., Nemesio Castillo, N. (2020). Physical intimate partner violence and controlling behavior in mexican university students and their attitudes toward social limits. Journal of Interpersonal Violence, 35(1-2), 403-425. DOI: https://doi.org/10.1177/0886260516681879

Estrada, C., Herrero, J. y Rodríguez, F. J. (2012). La red de apoyo en mujeres víctimas de violencia contra la pareja en el estado de Jalisco (México). Universitas Psychologica, 11(2), 523-534. Dor: https://doi.org/10.11144/Javeriana.upsy11-2.ramv

Ferrer, P. V. et al. (2006). La violencia contra las mujeres en la pareja: creencias y actitudes en estudiantes universitarios(as). Psicothema, 18(3), 359-366. Recuperado de http://www.psicothema.com/pdf/3223.pdf

Franco, F. P. y Orozco, M. (2019). Participación y movilización en la resistencia y defensa del territorio pacífico colombiano: Experiencias de la organización "Sé quién soy” y del Consejo de Comunidades Negras La Plata-Bahía Málaga. En P. Paño, R. Rébola y M. Suárez (comps.). Procesos y Metodologías Participativas Re- 
flexiones y experiencias para la transformación social (pp. 280-298). Buenos Aires, Argentina: Clacso-Udelar.

Fredman, S. (2016). Substantive equiality revisited. International Journal of Constitutional Law, 14(3), 712-738. Dor: https://doi.org/10.1093/icon/mow043

Frías, S. M. y Agoff, M. C. (2015). Between support and vulnerability: Examining family support among women victims of intimate partner violence in Mexico. Journal of Family Violence, 30(3), 277-291. DoI: https://doi.org/10.1007/s10896-0159677-y

García, F. et al. (2014). Violencia en la pareja, apoyo social y bienestar psicológico en adultos jóvenes. Ajayu. Órgano de Difusión Científica del Departamento de Psicología de la Universidad Católica Boliviana "San Pablo", 12(2), 246-265. Recuperado de http://www.scielo.org.bo/pdf/rap/v12n2/v12n2a5.pdf

Gobierno de México. Instituto Nacional de las Personas Adultas Mayores (s. f.). Importancia de las redes de apoyo social para las personas mayores. Recuperado de https://www.gob.mx/inapam/articulos/importancia-de-las-redes-de-apoyo-social -para-las-personas-mayores

Gobierno de México. Secretaría de Bienestar (2019). Informe anual sobre la situación de pobreza y rezago social 2019. Estado de Hidalgo. Recuperado de https://www. gob.mx/cms/uploads/attachment/file/432099/Hidalgo.pdf

González, H. y Fernández de Juan, T. (2010). Género y maltrato: violencia de pareja en los jóvenes de Baja California. Estudios Fronterizos, 11(22), 97-128. Recuperado de http://www.scielo.org.mx/scielo.php?script=sci_arttext\&pid=S0187$69612010000200004 \& \operatorname{lng}=\mathrm{es} \& \mathrm{nrm}=$ iso

Instituto Nacional de Estadística y Geografía (2019). Estadísticas a propósito del día internacional de la eliminación de la violencia contra la mujer (25 de noviembre). Datos nacionales. Recuperado de https://www.inegi.org.mx/contenidos/salade prensa/aproposito/2019/Violencia2019_Nal.pdf

Kelmendi, K. y Baumgartner, F. (2017). Exploring violence socialization and approval of intimate partner violence among University Students in Kosovo. Journal of Interpersonal Violence, 35(5-6), 1081-1107. DoI: https://doi.org/10.1177/0886260517692336

Lagarde, M. (1997). Identidad de género y derechos humanos. La construcción de las humanas. Recuperado de https://xenero.webs.uvigo.es/profesorado/marcela_lagarde/construccion_humanas.pdf

Lagarde, M. (2006). Pacto entre mujeres. Sororidad. Recuperado de http://pmayobre. webs.uvigo.es/textos/marcela_lagarde_y_de_los_rios/sororidad.pdf 
Lagarde, M. (2012). Enemistad y sororidad: hacia una nueva cultura feminista. Recuperado de http://www.oigahermanohermana.org/article-enemistad-y-sorori dad-hacia-una-nueva-cultura-feminista-104493332.html

Lagarde, M. (2013). Conferencia de Marcela Lagarde sobre "la sororidad". Recuperado de https://www.youtube.com/watch?v=8CKCCy6R2_g

Lazarevich, I., Irigoyen-Camacho, M. E.y Velázquez-Alva, M. C. (2017). Dating violence in Mexican college students: Evaluation of an educational workshop. Journal of Interpersonal Violence, 32(2), 183-204. Dor: https://doi.org/10.1177/0886260515585539

Li, L., Sun, I. Y. y Button, D. M. (2017). Tolerance for intimate partner violence: A comparative study of Chinese and American college students. Journal of Interpersonal Violence, 35(21). DoI: https://doi.org/10.1177/0886260517716941

Lomnitz, L. (1990). Redes de apoyo informales de intercambio en sistemas formales: un modelo teórico. Comercio Exterior, 3(40), 212-220. Recuperado de https://study lib.es/doc/7586941/redes-informales-de-intercambio-en-sistemas-formales

López-Cepero, J., Estrada-Pineda, C. y Chan-Gamboa, E. C. (2018). Effect of victimization and perceived support on maintenance of dating relationships among college students in Guadalajara, Mexico. Journal of Interpersonal Violence, 36(5-6). DOI: https://doi.org/10.1177/0886260518759057

Matud, M. P. et al. (2003). El apoyo social en la mujer maltratada por su pareja. International Journal of Clinical and Health Psychology, 3(3), pp.439-459. Recuperado de https://www.redalyc.org/articulo.oa?id=33730302

Morales, D. N. y Rodríguez, D. V. (2016). Experiencias de violencia en el noviazgo de mujeres en Puerto Rico. Revista Puertorriqueña de Psicología, 23(1), pp. 57-90. Recuperado de http://www.ojs.repsasppr.net/index.php/reps/article/view/199/199

Nordin, K. (2019). A bruise without a name: Investigating college student perceptions of intimate partner violence terminology. Journal of Interpersonal Violence, 0 , 1-25. DoI: https://doi.org/10.1177/0886260519876723

ONU. Mujeres (2019). Organización de las Naciones Unidas. Poner fin a la violencia contra mujeres y niñas. Violencia por un compañero sentimental. Recuperado de http://interactive.unwomen.org/multimedia/infographic/violenceagainstwo men/es/index.html\#intimate-3

Páramo, M. A. y Arrigoni, F. (2018). Violencia psicológica en la relación de noviazgo en estudiantes universitarios mendocinos (Argentina). Archivos de Medicina, 18(2). DOI: https://doi.org/10.30554/archmed.18.2.2738.2018

Pugh, B., Li, L. y Sun, I. Y. (2018). Perceptions of why women stay in physically abusive relationships: A comparative study of Chinese and U. S. college students. Journal of Interpersonal Violence, 36(7-8). DoI: https://doi.org/10.1177/0886260518778264 
Rodríguez, H. R., Riovelasco, L. M. y Castillo, V. N. (2018). Violencia en el noviazgo, género y apoyo social en jóvenes universitarios. Escritos de Psicología, 11(1), 1-9. DoI: https://doi.org/10.5231/psy.writ.2018.2203

Rojas-Solís, J. (2013). Violencia en el noviazgo de universitarios en México: Una revisión. Revista Internacional de Psicología, 2, 1-32. DoI: https://doi. org/10.33670/18181023.v12i02.71

Ruiz, J., Expósito, F. y Bonache, H. (2010). Adolescent witnesses in cases of teen dating violence: an analysis of peer responses. The European Journal of Psychology Applied to Legal Context, 2(1), 37-53. Recuperado de https://dialnet.unirioja.es/serv let/articulo?codigo $=3112482$

Segato, R. (2003a). Las estructuras elementales de la violencia: contrato y estatus en la etiología de la violencia. En R. Segato, Las estructuras elementales de la violencia (pp. 131-148), Buenos Aires, Argentina: Universidad Nacional de Quilmes.

Segato, R. (2003b). Los principios de la violencia. En R. Segato, Las estructuras elementales de la violencia (pp. 253-261). Buenos Aires, Argentina: Universidad Nacional de Quilmes.

Spencer, C. M., Morgan, P. y Bridges, J. (2017). The elationship between approval of violence and intimate partner violence in college students. Journal of Interpersonal Violence, 36(1-2). DoI: https://doi.org/10.1177/0886260517731315

Stylianou, A. M., Counselman-Carpenter, E. y Redcay, A. (2018). My sister is the one that made me stay above water: How social supports are maintained and strained when survivors of intimate partner violence reside in emergency shelter programs. Journal of Interpersonal Violence, 36(7-8). DoI: https://doi. org/10.1177/0886260518816320

Sundborg, E. et al. (2015). To ask or not to ask: the hesitation process described by district nurses encoutering women exposed to intimate partner violence. Clinical Nursing, 26(15-16), 2256-2265. DoI: https://doi.org/10.1111/jocn.12992

Universidad Autónoma del Estado de Hidalgo (2017). Anuario Estadístico 2017. Recuperado de https://www.uaeh.edu.mx/informe/2017-2023/1/docs/1er_Informe_ 2018/content/Anuario_2017.pdf

Vázquez, G. V. y Castro, R. (2008). ¿Mi novio sería capaz de matarme? Violencia en el noviazgo entre adolescentes de la Universidad Autónoma Chapingo, México. Revista Latinoamericana de Ciencias Sociales, Niñez y Juventud, 6, 709-738. Recuperado de http://www.redalyc.org/articulo.oa?id=77360208

Vizcarra, L. M. y Póo, F. A. (2011). Violencia de pareja en estudiantes universitarios del sur de Chile. Universitas Psychologica, 10(1), 89-98. Recuperado de http://www. 
scielo.org.co/scielo.php?script=sci_arttext\&pid=S1657-92672011000100008\&lng= es\&nrm=is.

Wang, L. (2019). Education, perception factors, and prevention of intimate partner violence: empirical research on Chinese university students' perceptions and attitudes concerning intimate partner violence. Journal of Interpersonal Violence, 34(8), 1611-1632. DoI: https://doi.org/10.1177/0886260516652263

Weisz, A. N. et al. (2007). Informal helpers' responses when adolescents tell them about dating violence or romantic relationship problems. Journal of Adolescence, 30(5), 853-868. DoI: https://doi.org/10.1016/j.adolescence.2006.09.004

Wilson, J. M y Smirles, K. (2020). College students' perceptions of intimate partner violence: The effects of type of abuse and perpetrator gender. Journal of Interpersonal Violence, 0, 1-23. DoI: https://doi.org/10.1177/0886260520908025 